\title{
Sepse em gestação pré-termo: relato de caso
}

\author{
Sepse in pre-term pregnancy: case report
}

Sepsis de embarazo pre-plazo: informe del caso

Marina Lopes de Freitas Freire ${ }^{1 *}$, Thaís Oliveira Santos ${ }^{1}$, Allen Washington Duarte Magalhães ${ }^{1}$, Adriel Rayol Aragão ${ }^{1}$, Carla Alessandra Haber Bastos ${ }^{1,2,3}$, Eduarda Teixeira Braga Bastos ${ }^{4}$, Marcello Oliveira Santos ${ }^{4}$, Aliandro Willy Duarte Magalhães ${ }^{4}$, Natália Tavares Carvalho ${ }^{1}$, Israela Cristine Pereira Marinho ${ }^{1}$.

\section{RESUMO}

Objetivo: Relatar um caso de sepse em gestação pré-termo, ocorrido em um hospital de ensino e pesquisa, referência no atendimento à gestante de alto risco, no Estado do Pará. Detalhamento do caso: Paciente do sexo feminino, 26 anos, primigesta, portadora de Artrite Reumatoide, com Idade Gestacional de 32 semanas e 3 dias com queixa de lombalgia e dor em baixo ventre. O quadro evoluiu com polaciúria, Giordano positivo a direita, colo entreaberto e perda do tampão mucoso. No segundo dia de internação a paciente apresentou taquicardia e após a realização da ultrassonografia obstétrica foi constatado a morte fetal intrauterina. Após avaliação, e posterior piora do quadro, foi prescrito novo de antibiótico e mudança para leito em unidade de terapia intensiva, assim como a realização da interrupção cirúrgica da gravidez. Considerações finais: Visto à rápida evolução do quadro de sepse, e ao difícil diagnóstico do quadro devido as mudanças fisiológicas da gravidez, ressalta-se a importância da agilização diagnóstica e pronta instituição terapêutica, a fim de se evitar as principais complicações do quadro, como mortalidade materno-fetal e prematuridade.

Palavras-chave: Sepse, Gravidez de alto risco, Mortalidade fetal.

\begin{abstract}
Objective: To report a case of sepsis in preterm pregnancy, which occurred in a teaching and research hospital, a reference in the care of high-risk pregnant women in the State of Pará. Case details: Female patient, 26 years old, firstborn, with rheumatoid arthritis, with gestational age of 32 weeks and 3 days complaining of backache and lower abdominal pain. The condition evolved with polyuria, positive Giordano on the right, half-open uterine lap, and loss of mucous plug. On the second day of hospitalization, the patient presented tachycardia and after obstetric ultrasonography, intrauterine fetal death was observed. After evaluation and subsequent worsening of the condition, a new antibiotic and a change of intensive care unit were prescribed, as well as surgical termination of pregnancy. Final considerations: Since of the rapid evolution of sepsis and the difficult diagnosis because the physiological changes of pregnancy, the importance of rapid diagnosis and prompt therapeutic institution is emphasized in order to avoid the main complications, such as maternal-fetal mortality and prematurity.
\end{abstract}

Key words: Sepsis, High-risk pregnancy, Fetal mortality.

\footnotetext{
${ }^{1}$ Centro Universitário Metropolitano da Amazônia (UNIFAMAZ), Belém - PA.

${ }^{*}$ E-mail: marinafreitasfreire@gmail.com

${ }^{2}$ Universidade do Estado do Pará (UEPA), Belém - PA.

${ }^{3}$ Santa Casa de Misericórdia do Pará, Belém - PA.

${ }^{4}$ Centro Universitário do Estado do Pará (CESUPA), Belém - PA.
}

SUBMETIDO EM: 2/2020

ACEITO EM: 3/2020

PUBLICADO EM: 6/2020 


\section{RESUMEN}

Objetivo: Informar un caso de sepsis en el embarazo prematuro, que ocurrió en un hospital de enseñanza e investigación, una referencia en la atención de mujeres embarazadas de alto riesgo en el Estado de Pará. Detalles del caso: Paciente de 26 años, primípara, con artritis reumatoide, con una edad gestacional de 32 semanas y 3 días que se queja de dolor lumbar y dolor en la parte inferior del abdomen. La condición evolucionó con polaciuria, Giordano positivo a la derecha, cuello medio abierto y pérdida del tapón mucoso. En el segundo día de hospitalización, la paciente presentó taquicardia y después de realizar una ecografía obstétrica, se observó la muerte fetal intrauterina. Después de la evaluación y el posterior empeoramiento de la afección, se prescribió un nuevo antibiótico y un cambio a la unidad de cuidados intensivos, así como la interrupción quirúrgica del embarazo. Consideraciones finales: En vista de la rápida evolución de la sepsis y del difícil diagnóstico de la afección debido a los cambios fisiológicos del embarazo, se enfatizala importancia de acelerar el diagnóstico y la pronta institución terapéutica, para evitar las principales complicaciones de la afección, como la mortalidad materna-fetal y prematuridad.

Palavras clave: Sepsis, Embarazo de alto riesgo, Mortalidade fetal.

\section{INTRODUÇÃO}

A sepse é definida como uma disfunção orgânica potencialmente fatal causada por uma resposta imune desregulada a uma infecção. Para a operacionalização clínica, a disfunção orgânica pode ser representada por um aumento do score sequencial de avaliação de falha de órgão (SOFA). O choque séptico pode ser definido como um subconjunto de sepse no qual anormalidades circulatórias, celulares e metabólicas, particularmente profundas estão relacionadas a um risco maior de mortalidade do que com sepse isolada (SINGER M, et al., 2016).

A sepse materna, segundo a Organização Mundial de Saúde (OMS), é uma ameaça à vida, condição definida como disfunção orgânica resultante de uma infecção durante a gravidez, parto, pós-aborto ou período pós-parto. Dados de 2006 a 2008 revelaram uma incidência de morte materna relacionada a sepse de 1,13 mortes/100 mil gestantes, se comprados com os índices de 2003 a 2005 (0,85 mortes/100 mil gestantes), observa-se o grande aumento desses casos.

Em relação a mortalidade de gestantes por choque séptico as taxas são muito inferiores se comparadas a população em geral. Essa menor taxa de mortalidade pode ser explicada pelo fato da gestante possuir alguns fatores protetores, tais como: menor presença de comorbidades associadas, possuírem idades mais jovens e sítios de infecção de fácil identificação (na população obstétrica o sítio urogenital é o foco de maior parte dos casos de sepse), facilitando o diagnóstico e consequentemente o tratamento e a exploração cirúrgica, quando necessário (WORLD HEALTH ORGANIZATION, 2017; CORDIOLI LC, et al., 2013).

As bactérias Gram-negativas constituem os vermes, geralmente, mais envolvidos na ocorrência desses quadros, podendo evoluir a sepse de origem polimicrobiana (bactérias anaeróbias, Gram-positivas e fungos estão presentes na flora urogenital).

Outros fatores que predispõem a sepse na gravidez são: cesariana, inadequação do acompanhamento pré-natal e ausência de políticas de abortamento legalizado, indução de aborto, rompimento prematuro de membrana ovalares, além de condições inerentes a população obstétrica como: vagina e endocervix que abrigam inúmeras bactérias, propagação de germes durante o parto normal, possível infecção de sítio cirúrgico durante cesárea e/ou episiotomia, múltiplos exames genitais periparto, bem como diminuição da resposta imune celular mediada (CORDIOLI LC, et al., 2013).

Durante a gravidez, diversas alterações fisiológicas no organismo materno levam a maior predisposição à infecção urinária e suas complicações. A estase urinária secundária, a compressão do ureter pelo útero gravídico e o relaxamento da musculatura por ação da progesterona favorecem o surgimento de bacteriúria, sendo um fator de risco importante para o desenvolvimento de pielonefrite e suas complicações (GUERRA GVQL, et al., 2012). 
A sepse urinária é considerada como um problema urinário comum na gestação, e é caracterizada pela presença de agentes infecciosos que colonizam, invadem e se propagam pelo trato urinário, sendo classificada segundo a sua localização em sepse urinária baixa e alta. Esta infecção ocorre entre 17 a 20\% das gestações e associa-se a complicações como rotura prematura de membranas ovulares, parto prematuro, coriomnionite, febre no pós-parto, sepse materna e infecção neonatal, além de hipertensão/pré-eclâmpsia, anemia e endometrite, destacando-se também nas complicações perinatais, recém-nascido de baixo peso, ruptura prematura de membranas amnióticas, a restrição de crescimento intraútero, a paralisia cerebral/retardo mental e o óbito perinatal (SOUZA JPL e MENDES DRG, 2015).

$\mathrm{Na}$ gravidez, a infeção materna aguda pode estimular a contractilidade uterina, induzir modificações cervicais e conduzir a um parto prematuro. Por outro, as modificações fisiológicas da gravidez podem condicionar a capacidade de adaptação materna e de resposta à doença. E por último, o risco de infeção fetal, de efeitos hemodinâmicos ou inflamatórios fetais secundários à doença e ao tratamento maternos, 0 risco de encefalopatia neonatal e de paralisia cerebral não devem ser desprezados (TORRES R, et al., 2015).

Sabe-se que, no Brasil, a assistência pré-natal ainda é um desafio para o Sistema Único de Saúde (SUS). A despeito da melhor assistência obstétrica oferecida às gestantes, taxas significativas de morbimortalidade materna ainda podem ser observadas durante a gravidez e o parto. Dentro deste contexto, um indicador importante de morbidade materna é a necessidade de transferência de paciente obstétrica para a Unidade de Terapia Intensiva (UTI).

Um boletim divulgado em 2010 pela OMS, apontou que a incidência de gestantes brasileiras que necessitaram de internação em UTI foi de 2,1\%. Sendo as síndromes hipertensivas, os quadros hemorrágicos e a sepse de causa obstétrica as principais causas dessas internações. Foi-se observado que as barreiras de acesso a não realização do pré-natal, principalmente em mulheres das regiões Norte e Nordeste do país, se deu em decorrência do menor nível educacional dessa população. Fato este que pode ser entendido como lacunas assistenciais existentes em grandes territórios, assim como as próprias limitações impostas pela baixa escolaridade (REISDORFER SM, et al., 2013; SAINTRAIN SV, et al., 2016).

O estudo teve como objetivo relatar um caso de sepse em gestação pré-termo, ocorrido em um hospital de ensino e pesquisa, referência no atendimento à gestante de alto risco, no Estado do Pará.

\section{RELATO DE CASO}

Paciente do sexo feminino, 26 anos, primigesta, portadora de Artrite Reumatoide, com Idade Gestacional de 32 semanas e 3 dias - pela data da última menstruação (DUM) - procurou o atendimento de uma maternidade de médio e alto risco de Belém/PA na noite do dia 16/08/2018, apresentando lombalgia e dor em baixo ventre. Negava sintomas urinários como disúria e polaciúria. Apresentava-se em bom estado geral, consciente e orientada, acianótica, anictérica, afebril, hidratada e eupneica. Ao exame obstétrico encontravase com dinâmica uterina (DU) ausente, altura uterina (AU) de $32 \mathrm{~cm}$, batimentos cardíacos fetais (BCF) de $148 \mathrm{bpm}$ e colo grosso, posterior e impérvio. Na conduta foram solicitados hemograma, Veneral Disease Research Laboratory (VDRL), tipagem sanguínea, urina, ultrassom obstétrico (USGO) com Doppler e foram prescritos sintomáticos para dor. Sendo a paciente internada na enfermaria do serviço.

Durante reavaliação, no dia 17/08/2018, a paciente evoluiu com lombalgia de forte intensidade com irradiação para baixo ventre com início há 24 horas, associado, agora, a polaciúria, Giordano positivo a direita, colo entreaberto e perda do tampão mucoso. Aos exames solicitados apresentava anemia - hemoglobina de $9 \mathrm{~g} / \mathrm{dL}$ (valor de referência: $11,5-14,9 \mathrm{~g} / \mathrm{dL}$ ), leucócitos de 8.496/ $\mathrm{mm}^{3}$ (valor de referência: 2.883 $9.969 / \mathrm{mm}^{3}$ ), porém apresentava $9 \%$ de bastões e $75 \%$ de segmentados, plaquetas de $80970 / \mathrm{mm}^{3}$ (valor de referência: $137.881-344.744 / \mathrm{mm}^{3}$ ), VDRL não reagente, e tipo sanguíneo O positivo. Ao USGO com Doppler apresentava-se com 33 semanas, placenta grau 2, índice de líquido amniótico (ILA) 15,4 cm, Doppler sem alterações. Apresentava urina turva com presença de proteinúria e hematúria. Foi solicitado, então, a internação, novo hemograma, Ultrassom de Vias Urinarias (USGVU), novo USGO com Doppler. Além disso, devido a presença de proteinúria e hematúria no exame de urina, foi suspeitado e tratado para uma possível Pielonefrite com ceftriaxona. 
No segundo dia de internação a paciente apresentava o mesmo quadro associado a taquicardia (frequência cardíaca de 124bpm) e o resultado do USGO mostrava morte fetal intrauterino. Os demais exames ainda não estavam prontos. Por causa da taquicardia e do hemograma com desvio, foi aberto o protocolo para Sepse, solicitado a avaliação da clínica médica e foi prescrito indução do parto com misoprostol e orientação ao paciente e família. Já no terceiro dia de internação a paciente evoluiu com dispneia, taquipneia, taquicardia, constipação, dor pélvica, dor abdominal e disúria. Ao exame foi encontrado abolição do murmúrio vesicular em base direita, saturação de $82 \%$ e edema.

Desse modo, foi prescrito a mudança do antibiótico para o Piperaciclina sódica + tazobactam sódico, além da utilização de cloridrato de metoclopramida e glicerina via retal. Além disso, foi solicitado suporte ventilatório, laboratório de urgência, leito na UTI e TC de abdome total para avaliar um possível foco abdominal da sepse. Foi realizado, também, a interrupção cirúrgica da gravidez por causa do quadro apresentado e por estar no sétimo ciclo de misoprostol. A paciente evoluiu de maneira satisfatória, tendo alta hospitalar após 10 dias.

\section{DISCUSSÃO}

O presente estudo procura ressaltar a importância da identificação imediata e da rápida intervenção na sepse durante a gestação. Embora seja uma complicação rara, a sepse é uma síndrome que apresenta crescimento em sua incidência em todo mundo e é fatal tanto para o feto quanto para mãe, sendo uma das cinco principais causas de mortalidade materna (ANTHONY M, et al., 2018; ZASTROW JB, et al., 2018). Sabendo disso, é importante encontrar rapidamente o foco da infecção para estabelecimento da antibioticoterapia (ANTHONY M, et al., 2018).

Por ser tão fatal, foram pesquisadas as principais causas de sepse para ajudar na rápida busca etiológica e, consequentemente, no rápido manejo. Sendo assim, separaram a sepse na gestação em dois grupos de acordo com as causas: as não obstétricas e obstétricas. Estão inclusos no primeiro grupo as infecções do trato urinário, pneumonias adquiridas na comunidade, apendicite, coleciste, vírus da imunodeficiência humana (HIV) e malária. Já as obstétricas englobam a corioamnionite, tromboflebite pélvica séptica e aborto infectado, endometrite pós-parto, infecção de episiotomia, dentre outras. Simplificando, a sepse de origem obstétrica, em geral, tem origem polimicrobiana, com prevalência de bactérias Gram-negativas e anaeróbios (ZASTROW JB, et al., 2018).

Dentre todas as causas citadas e compatível com o relato de caso descrito, a Infecção do trato urinário (ITU) é uma das infecções mais comuns na gestação, sendo a principal causa de sepse no período gestacional.13 Esse fato é ocasionado por alterações funcionais, anatômicas e hormonais na mulher gravídica. Uma anamnese e semiologia clínica adequada podem apontar dor abdominal ou pélvica, leucorréia purulenta e de odor fétido, dor em flancos, hiperemia mamária e disúria, sinais e sintomas que são sugestivos de infecção. Assim como, a avaliação do bem-estar fetal, deve ser prontamente instituída, visto que a taquicardia fetal pode ser um sinal precoce de sepse (CORDIOLI LC, et al., 2013; De MATOS, et al., 2019).

Dentre as mudanças fisiológicas gravídicas, podemos destacar a leucocitose, a plaquetopenia e a alcalose secundária à taquipneia, fazendo com que a interpretação dos resultados de exames laboratoriais seja feita de forma a considerar essas distinções. Com isso, pode-se dizer que o principal agente infeccioso, quando retratamos o foco urinário, é a E. coli e a ceftriaxona é o antibiótico mais utilizado para Pielonefrite de origem comunitária. Entretanto, o aumento da resistência bacteriana e a própria alteração fisiológica da gravidez podem limitar ainda mais o arsenal disponível (De MATOS, et al., 2019; BARRETO S, 2018).

Aliado a resistência, outros fatores que podem dificultar o manejo rápido da sepse são as modificações fisiológicas da gestação. Essas modificações tornam mais difíceis a interpretação de algumas alterações resultantes do quadro de infecção grave. Esse fato facilita diagnósticos equivocados e, consequentemente, leva ao atraso na abordagem mais adequada para o caso de sepse (ABASSI N, et al., 2014). Neste relato, a paciente não apresentou sinais e sintomas mais comumente associados com infecção, como febre ou hipotermia, hipóxia, hipotensão, e foi conduzida a partir dos sinais inespecíficos, como taquicardia e hemograma com desvio, o que retardou o diagnóstico e a conduta mais adequada de maneira precoce. 
Esta pesquisa demonstrou uma rápida evolução da Infecção Urinaria para o quadro de sepse, levando assim, para o quadro de disfunção orgânica materna e, provavelmente, para morte fetal. Sistemas de pontuação, para auxiliar no diagnostico precoce e tratamento em casos suspeitos, já foram criados, em virtude da rápida evolução dos quadros de sepse.

No entanto, ainda não encontraram um sistema amplamente validado para mulheres grávidas, pois, os preexistentes possuem uma grande variação tanto na sensibilidade quanto na especificidade. Por conta disso, atualmente, o diagnóstico de sepse na gravidez é feito com base na suspeita clínica associada as discussões recentes que deram maior ênfase nos sinais de disfunção orgânica (neurológico, cardiovascular, respiratório, gastrointestinal, urológico e hematológico) (ANTHONY M, et al., 2018).

A sepse materna constitui um desafio na prática clínica, visto que o diagnóstico é baseado na suspeita clínico, devido, em grande parte, as mudanças fisiológicas da gravidez dificultarem a interpretação de sinais e sintomas típicos. O presente caso clínico demonstrou uma paciente com sepse na gestação de possível foco urinário e com uma evolução insatisfatória, mesmo com a terapêutica adequada. Assim, ressalta-se a importância de agilizar o diagnóstico e, consequentemente, adequar o manejo, evitando as principais complicações relatadas da sepse (mortalidade materno-fetal e a prematuridade).

\section{REFERÊNCIAS}

1. ABBASI N, et al. Evaluation of obstetrical and fetal outcomes in pregnancies complicated by acute appendicitis. Archives of gynecology and obstetrics, 2014; 290(4): 661-7.

2. ANTHONY M, et al. Recognition and Treatment of Sepsis in Pregnancy. Journal of Midwifery \& Women's Health, 2018; 63(3).

3. BARRETO S. revalência de uropatógenos e perfil de sensibilidade aos antibacterianos em uroculturas de gestantes atendidas no Hospital Geral de Fortaleza. Fortaleza/Ceará, 2018

4. CORDIOLI LC, et al. Sepse e gravidez: sabemos tratar? Rev. Bras. Ter. Intensiva. 2013; 25(4):334-344.

5. DE MATOS KLA, et al. Sepse durante o período gestacional. Revista Eletrônica Acervo Saúde.2019; 11(17), e1166.

6. GUERRA GVQL, et al. Exame simples de urina no diagnóstico de infecção urinária em gestantes de alto risco. Rev. Bras. Ginecol. Obstet. 2012; 34(11): 488-93.

7. ILAS, 2016 Instituto Latino Americano De Sepse. Relatório ILAS [internet] 2017 Dic [citado em 13 de julho de 2017];

8. REISDORFER SM et al. Características clínicas de pacientes obstétricas admitidas em uma Unidade de Tratamento Intensivo Terciária: revisão de dez anos. Revista da AMRIGS. Porto Alegre, 2013; 57(1): 26-30.

9. SAINTRAIN SV, et al. Fatores associados à morte materna em unidade de terapia intensiva. Rev. Bras. Ter. Intensiva. 2016; 28(4): 397-404.

10. SINGER M, et al. The Third International Consensus Definitions for Sepsis and Septic Shock (Sepsis-3). JAMA. 2016; 315(8): 801-10.

11. SOUZA JPL, MENDES DRG. Prevalência de sepse urinária em gestantes da UBS/ESF do Cruzeiro do Sul. REVISA. 2015; 4(1): 40-51.

12. STATEMENT ON MATERNAL SEPSIS. 2017 World Health Organization.

13. TORRES R, et al. Sepsis maternal. Acta. Obstet. Ginecol. Port. 2015; 9(1): 65-72.

14. ZASTROW JB, et al. Sepse em gestantes atendidas em um hospital público de Curitiba - PR. Rev Soc Bras Clin Med. 2018;16(4): 208-11. 